\title{
RECUPERACIÓN ACELERADA EN CIRUGÍA COLORRECTAL LAPAROSCÓPICA ELECTIVA: DOS AÑOS DE EXPERIENCIA.
}

\section{ACCELERATED RECOVERY IN ELECTIVE LAPAROSCOPIC COLORECTAL SURGERY: TWO YEARS OF EXPERIENCE. \\ RECUPERAÇÃO ACELERADA EM CIRURGIA COLORRETAL LAPAROSCÓPICA ELETIVA: DOIS ANOS DE EXPERIÊNCIA.}

\author{
Reimondez Santiago ${ }^{1,2}$, Sgnorini Franco Jose ${ }^{1}$, Maldonado Pablo Sergio ${ }^{1}$, Marani Marcos \\ Agustin $^{1}$, Obeide Lucio Ricardo ${ }^{1}$, Rossini Alejandro Mario ${ }^{1}$ \\ Resumen:
}

1 Hospital Privado Universitario de Córdoba. Instituto Universitario de Ciencias Biomédicas de Córdoba, Córdoba capital, Argentina.

2 Email de contacto: santi rei@hotmail.com

\section{Conceptos clave:}

Los protocolos de recuperación acelerada han demostrado reducir la morbilidad perioperatoria y la estadía hospitalaria en pacientes sometidos a cirugía colorrectal laparoscópica.

\section{Que aporta este trabajo}

A partir de la adecuación de los postulados de los consensos internacionales a la realidad sanitaria local se ha logrado la confección y ejecución de un protocolo multidisciplinario de recuperación acelerada con resultados alentadores.
El desarrollo de los protocolos de recuperación acelerada en cirugía colorrectal ha revalorizado los cuidados que conforman la recuperación perioperatoria de los pacientes sometidos a cirugía. Se persigue una reducción del stress quirúrgico y por tanto alta precoz y menor morbilidad, sin embargo, la literatura aporta pocos resultados respecto a su aplicación en países en desarrollo. Objetivo: analizar los resultados a corto y mediano plazo de un protocolo de recuperación acelerada en un hospital de alta complejidad de nuestro medio. En el periodo comprendido entre enero 2015 y marzo 2017 se incluyeron pacientes de manera prospectiva y consecutiva con indicación de cirugía colorrectal laparocopica electiva y bajo estricto seguimiento según protocolo creado por la institución. Fueron excluidos pacientes mayores de 80 años, ASA IV, cirugías de urgencia y abordaje convencional. Se analizaron datos demográficos indicación de cirugía, tipo de intervención, estadía hospitalaria en días, complicaciones, readmisiones y reintervenciones a los 30 días de postoperatorio. Resultados: Fueron incluidos 64 pacientes con una edad media de 62 años. El promedio de internación en días fue de 4,9 , con $10,9 \%$ de reinternaciones y $4,7 \%$ de reintervenciones. El $69 \%$ de los pacientes no presento complicaciones, registrándose 5 complicaciones mayores $(8 \%)$. Conclusión: A partir de la adecuación de los lineamientos internacionales a nuestra realidad sanitaria, es factible la creación de un protocolo de recuperación acelerada aplicable en nuestro medio, con baja tasa de complicaciones y alta precoz.

Palabras claves: recuperación acelerada, laparoscópico, cáncer de colon, fastrack.

\section{Abstract:}

Accelerated recovery protocols in colorectal surgery have enhanced the perioperative cares optimizing recovery in this group of patients. A reduction in surgical stress and therefore early hospital discharge and lower morbidity is pursued, however, the literature offers few outcomes regarding its application in developing countries.

Objective: to analyze short- and medium-term outcomes of the application of an accelerated recovery protocol in a terciary care hospital in Argentina. Methods: In the period between January 2015 and March 2017 patients were included prospectively and consecutively with indication of elective laparoscopic colorectal surgery and under strict follow-up according to the protocol created by the institution. Patients older than 80 years, ASA IV, emergency surgeries and conventional approach were excluded. We analyzed demographic data, diagnosis of surgery, type of intervention, hospital stay, complications, readmissions and reinterventions at 30 postoperative days. Results: Sixty-four patients with a mean age of 62 years were included. The mean hospitalization was 4.9 days, with $10.9 \%$ readmissions and $4.7 \%$ of reinterventions. We recorded $69 \%$ of the patients whit not complications at all and 5 major complications (8\%) Conclusion: Based on the adaptation of the international guidelines to our health reality, it is feasible to create an accelerated recovery protocol applicable in our country, with a low complication rate and early discharge.

Key words: accelerated recovery, laparoscopic, colon cancer, fastrack

\section{Resumo:}

O desenvolvimento de protocolos de recuperação acelerada em cirurgia colorretal revalorizou os cuidados que compõem a recuperação perioperatória de pacientes submetidos à cirurgia. Busca-se uma redução no estresse cirúrgico e, portanto, alta morbidade precoce e menor, no entanto, a literatura fornece poucos resultados em relação à sua aplicação em países em desenvolvimento. Objetivo: analisar os resultados de curto e médio prazo de um protocolo de recuperação acelerada em um hospital de alta complexidade do nosso ambiente. No período de janeiro de 2015 a março de 2017 , os pacientes foram incluídos de forma prospectiva e consecutiva, com indicação de cirurgia colorretal laparocópica eletiva e com acompanhamento rigoroso, de acordo com o protocolo criado pela instituição. Foram excluídos pacientes com idade superior a 80 anos, ASA IV, cirurgia de emergência e abordagem convencional. Os dados demográficos foram analisados indicando cirurgia, tipo de intervenção, internação hospitalar em dias, complicações, readmissões e reintervenções aos 30 dias de pósoperatório. Resultados: foram incluídos 64 pacientes com idade média de 62 anos. A internação média em dias foi de 4,9, com 10,9\% dos reembolsos e $4,7 \%$ das reintervenções. $69 \%$ dos pacientes não apresentaram complicações, registrando 5 complicações maiores (8\%). Conclusão: A partir da adaptação das diretrizes internacionais à nossa realidade em saúde, é possível criar um protocolo de recuperação acelerada aplicável em nosso ambiente, com baixo índice de complicações e alta precocidade.

Palavras-chave: recuperação acelerada; laparoscópica; câncer de cólon; fastrack. 


\section{Introducción}

En las dos décadas pasadas, se desarrollaron los programas de recuperación acelerada como parte de la evolución en los cuidados perioperatorios de los pacientes sometidos a cirugía colorectal1-3. Estos programas se centran en la mejora de aspectos individuales de todo el período perioperatorio a través de un trabajo multidisciplinario y de medicina basada en la evidencia. El objetivo es acelerar la recuperación postoperatoria disminuyendo el stress quirúrgico a fin de disminuir la morbilidad y la estadía hospitalaria.

Los últimos consensos internacionales han demostrado que los viejos paradigmas como la preparación colónica, invasiones, reposo en cama y ayunos prolongados deben ser reemplazados y completados por la educación del paciente, cambios en el manejo anestésico y analgésico, la optimización nutricional y la movilización precoz entre otros ${ }^{4}$. La evidencia científica ofrece resultados que favorecen esta nueva forma manejo multidisciplinario ${ }^{5}$. Sin embargo existe escasa bibliografía regional que avale su implementación en centros de nuestra comunidad ${ }^{6}$.

El objetivo del presente trabajo es analizar los resultados a corto y mediano plazo de la aplicación de un protocolo de recuperación acelerada en cirugía colorrectal laparoscópica electiva en un centro de alta complejidad de nuestro medio.

\section{Materiales y metódos}

Se incluyeron todos los pacientes con diagnóstico de patología colorrectal con necesidad de cirugía de forma prospectiva, de manera consecutiva, no randomizada en el período comprendido entre enero 2015 y marzo 2017.

Se consideró criterio de exclusión la edad mayor de 80 años, la indicación de abordaje convencional, pacientes con comorbilidad cardiopulmonar significativa (ASA 4 o con necesidad de postoperatorio en unidad cerrada), estadío IV de enfermedad

tumoral maligna, alteraciones cognitivas o inadecuado acompañamiento social y pacientes que se negaron a formar parte a través del consentimiento informado. No fue considerado como tal la realización de ostomía.

La aplicabilidad del protocolo fue evaluada en casos pilotos hasta la confección final del mismo basado en los últimos consensos internacionales y bajo la supervisión y aceptación del comité de ética de la institución. Los principales postulados se resumen en la tabla 1.

\section{Tabla 1- Protocolo creado y aprobado por la institución.}

\section{PREOPERATORIO}

Evaluación preoperatoria en consultorio y explicación detallada de los pilares del protocolo

Educación con información escrita e ilustrativa y especificación de objetivos diarios

Evaluación del acompañamiento social y planificación de alta según criterios preestablecidos

Evaluación y asistencia nutricional por parte del equipo especializado de la institución

Admisión del paciente a la institución el mismo día de la cirugía

Abolición de ayuno prolongado, con ingesta de sólidos hasta 6 horas previas y líquidos claros hasta 2 horas

Ingesta de bebida rica en carbohidratos. Cada $100 \mathrm{ml}$ de gatorade ${ }^{\circledR} 7$ gr de polimerosa. Ingesta de $800 \mathrm{ml}$ el día previo y 200 $\mathrm{ml}$ dos horas antes de la cirugía

Abolición de preparación mecánica colónica en cirugía de colon derecho. Preparación reducida y domiciliaria con colectomías izquierda y rectales con barex kit $^{\circledast}$

INTRAOPERATORIO

Profilaxis con cefalosporina de primera generación + metronidazol

Analgesia epidural (torácica media o baja)

Drogas anestésicas de vida media corta

Prevención de hipotermia con manta térmica desde inducción

Fluidoterapia conservadora evitando la hiperhidratación

Antieméticos profilácticos durante la inducción

Dexametasona $8 \mathrm{mg}$ endovenosa en inducción

Abolición de sonda nasogástrica

Abolición de vía central

POSTOPERATORIO

Día de la cirugía

Goma de mascar en recuperación

Movilización precoz al menos dos horas diarias

Ingesta temprana de líquidos con suplementación vía oral (4 horas posterior a la intervención)

Antieméticos reglados de manera profiláctica

Analgesia con Antiinflamatorios no esteroideos vía oral, abolición de opioides

Primer día postoperatorio

Retiro de catéter urinario

Retiro de fluidos endovenosos 


\section{Pasaje de medicación a vía oral \\ Movilización activa al menos 6 horas diarias \\ Ingesta de líquidos y suplementación vía oral \\ Segundo día postoperatorio \\ Se incorpora dieta blanda \\ Movilización al menos 6 horas diarias \\ Tercer día postoperatorio \\ ALTA según criterios previamente establecidos}

\section{Tabla $N^{0}$ 1: Protocolo creado y aprobado por la institución.}

El alta fue planificada al tercer día de postoperatorio según cumplimiento de criterios previamente establecidos (tabla 2). Se realizó llamada telefónica a familiar o paciente a las 48 horas del alta.

\begin{tabular}{l}
\hline Criterios de alta \\
\hline Tolerancia vía oral a dieta semisólida \\
Deambulación completa \\
Buen manejo del dolor con analgesia vía oral \\
Eliminación de gases y/o deposición \\
Conformidad del paciente con el alta institucional \\
Soporte ambulatorio adecuado \\
\hline
\end{tabular}

\section{Tabla $N^{\circ}$ 2: Criterios de alta institucional}

Los procedimientos fueron llevados a cabo por dos cirujanos del servicio especialistas en cirugía colorrectal. No se modificó la técnica quirúrgica respecto a intervenciones previas a la implementación del programa. Todos los especialistas intervenientes recibieron cursos de formación en programas de recuperación acelerada. No se incluyó servicio de enfermería de dedicación exclusiva.

Todos los aspectos del protocolo fueron explicados en detalle a los pacientes, previo a la incorporación de los mismos. Se entregó un informe escrito y detallado con instrucciones para su correcta preparación y adecuación. El mismo incluía los postulados sobre la movilización activa, información nutricional y manejo anestésico, específicamente elaborados por cada servicio interviniente.

No se utilizó preparación mecánida de rutina para colectomías derechas. En el caso de colectomías izquierdas o resecciones rectales se realizó la misma de forma ambulatoria y reducida, con politielinglicol en polvo de disolución en 1 litro de agua, con ingesta 48 y 24 horas previas a la cirugía y la incorporación de 4 comprimidos de bisacodilo el día previo a la intervención (Barex

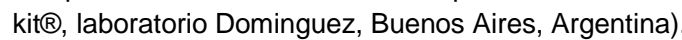

Cada paciente recibió instrucciones respecto a movilización precoz, con dos horas de movilización activa el mismo día de la cirugía y hasta 6 horas los días restantes, a cargo del fisioterapeuta de la institución.

La ingesta precoz consistió en líquidos claros desde el mismo día de la intervención, con comienzo luego de 4 horas de su finalización, acompañado de suplementación vía oral (Fresubin ${ }^{\circledR}$ lata, laboratorios Fresenius Kabi, Buenos Aires, Argentina). Dieta blanda fue incorporada al segundo día postoperatorio.
Todos los pacientes fueron educados para cumplir con ingesta de preparación rica en carbohidratos hasta dos horas antes de la intervención quirúrgica.

Fueron analizados datos demográficos, indicación de cirugía, tipo de intervención, estadía hospitalaria en días, complicaciones, readmisiones y reintervenciones en el período comprendido en los primeros 30 días de postoperatorio, independientemente de la causa. Las complicaciones fueron registradas según criterios de clasificación de Clavien Dindo ${ }^{7,8}$, considerándose únicamente el mayor grado registrado por el paciente. Se consideró complicación mayor a los grados III, IV y V.

Para el diagnóstico de fistula anastomótica se consideraron los criterios de Bruce $^{9}$ y Rahbari ${ }^{10}$. Se consideró como tal la presencia de peritonitis localizada o generalizada, la salida por herida quirúrgica o drenaje de pus o material fecaloideo, los abscesos intrabdominales y las infecciones pélvicas. La fiebre en ausencia de los signos o síntomas descriptos o con estudio de imagen no confirmatoria no fue considerada como tal. Ante la sospecha de fistula se realizó tomografía axial computada con contraste endovenoso en todos los pacientes. Dicho estudio no fue realizado como método de rutina en ausencia de sospecha de complicación.

\section{ANALISIS ESTADISTICO}

Las variables numéricas se expresan como media y rango y las categóricas como porcentaje según corresponda. Los datos fueron analizados con el programa spss $21 \AA$.

\section{Resultados}

En el periodo comprendido entre enero 2015 y marzo 2017, 64 pacientes con diagnóstico de patología colónica o rectal fueron intervenidos bajo abordaje laparoscópico electivo y seguimiento con protocolo de recuperación acelerada. En el mismo período se excluyeron 60 pacientes por comorbilidades significativas con necesidad de postoperatorio en unidad de cuidados intensivos, cirugías de emergencia o por la negación a partir del consentimiento informado.

Los datos demográficos, indicación y tipo de cirugía se resumen en la tabla 3. La edad media fue de 62 años (rango 39-80), $53 \%$ pacientes femeninos. El $90 \%$ fue intervenido por patología maligna. El $70 \%$ de los pacientes tenia clasificación de ASA II.

La media de alta en días fue de 4,9 (rango 3-26), con 10,9\% de reinternaciones y $4,7 \%$ de reintervenciones (tabla 4). El $69 \%$ de los pacientes no presento complicaciones, registrándose un $8 \%$ de complicaciones mayores según clasificación de Clavien Dindo. Se registraron 5 pacientes con diagnostico de fistula anastomótica $(7,8 \%), 2$ de los cuales requirieron reintervención quirúrgica y 1 óbito por infección respiratoria que requirió reinternacion en unidad de cuidados intensivos. 
Tabla N³: Datos demográficos, indicación y tipo de intervenciones.

\begin{tabular}{|c|c|c|}
\hline Variables & $\mathbf{N}$ & $\%$ \\
\hline Edad (media; rango) & $62 ; 39-79$ & \\
\hline$\overline{I M C}$ (media) & 27 & \\
\hline \multicolumn{3}{|l|}{ Genero } \\
\hline Masculino & 30 & 47 \\
\hline Femenino & 34 & 53 \\
\hline \multicolumn{3}{|l|}{ Score ASA } \\
\hline $\bar{I}$ & 5 & 8 \\
\hline II & 45 & 70 \\
\hline III & 14 & 22 \\
\hline \multicolumn{3}{|l|}{ Indicación de cirugía } \\
\hline Malignidad & 58 & 91 \\
\hline Patología benigna & 6 & 9 \\
\hline \multicolumn{3}{|l|}{ Tipo de cirugía } \\
\hline Colectomía derecha & 20 & 31 \\
\hline Colectomía izquierda & 13 & 20 \\
\hline $\mathrm{RAB}^{*}$ & 18 & 28 \\
\hline Colectomía segmentaria & 8 & 13 \\
\hline Amputación abdomino-perineal & 1 & 1,5 \\
\hline Retransitación de Hartmann & 3 & 5 \\
\hline Colectomía subtotal o total & 1 & 1,5 \\
\hline Tiempo quirúrgico (media; rango) & $146 ; 64-500$ & \\
\hline * Resección anterior baja & & \\
\hline
\end{tabular}

Tabla 4:- Resultados quirúrgicos y complicaciones.

\begin{tabular}{lc|c}
\hline Variable & N & $\%$ \\
\hline Alta (media; rango) & 4,$9 ; 3-29$ & \\
\hline Reinternación & 7 & 11 \\
\hline Días reinternación (media; rango) & $12 ; 3-29$ & \\
\hline Reintervención & 3 & 4,7 \\
\hline Fistula anastomótica & 5 & 7,8 \\
\hline Clavien Dindo & 44 & 69 \\
\hline No & 4 & 6 \\
\hline I & 11 & 17 \\
\hline II & 2 & 3,2 \\
\hline III & 2 & 3,2 \\
\hline IV & 1 & 1,6 \\
\hline V & &
\end{tabular}

\section{Discusión}

Este trabajo reporta los dos primeros años de experiencia en cirugía colorectal laparoscópica bajo seguimiento de un estricto protocolo de recuperación acelerada en un hospital de alta complejidad de la ciudad de Córdoba, Argentina.

Utilizando un abordaje multidisciplinario y un estructurado protocolo de seguimiento postoperatorio basado en los últimos consensos disponibles ${ }^{4}$, se logró el alta institucional del $50 \%$ de los pacientes en el tercer día postoperatorio y el $75 \%$ antes del cuarto día. Resultados muy similares a los obtenidos por series de casos previamente publicadas ${ }^{11,12}$. El protocolo fue estipulado con alta al tercer día según criterios preestablecidos, en correspondencia con el trabajo de Andersen et all $^{13}$ que demostró que altas más tempranas implican un aumento del índice de readmisión, probablemente debido a factores sociales y a la educación menos exaustiva de los pacientes.

El $69 \%$ de los pacientes fueron externados sin complicaciones, siendo un $8 \%$ el porcentaje de complicaciones mayores según la clasificación de Clavien Dindo ${ }^{7,8}$, similar a otras series y mataanalisis ${ }^{11,12,14}$. Se registro un $4,7 \%$ de reintervenciones, todas ellas debidas a fistula anastomotica. Respecto a esta última, se realizo diagnóstico de fistula anastomotica en 5 pacientes. Dos de ellos requieron reintervención por lo que se clasificarían como fistula mayor según la clasificación de Bruce o Fistula grado C según Rahbari. Los casos restantes fueron tratados con antibioticoterapia de amplio espectro y con drenaje percutáneo del mismo según fuese necesario, sin complicaciones posteriores y externados con buena evolución clínica. Si bien estos datos son similares al metaanalisis de Aarts et all ${ }^{12}$, esta cifras superan a las presentadas por series de casos similares ${ }^{5,11,14}$. Sin embargo ninguno de los trabajos citados describen los criterios diagnósticos según los estándares internacionales aceptados en la actualidad. Por otro lado, los autores consideran que en series de escaso número de pacientes, la aparición de eventos adversos afectan notoriamente el promedio, pudiendo corresponder a factores meramente azarosos. Se registro un óbito por infección respiratoria aguda con necesidad de reinternacion, lo que corresponde al $1,56 \%$ de la casuística presentada. Si bien estos valores son dispares según la serie analizada $^{5,15}$, registramos dicha complicación en el primer año de experiencia sobre un total de 42 casos (2\%). La mejora en los cuidados perioperaotorios y la búsqueda de mejores estándares de calidad nos permiten inferir un descenso porcentual a medida que mas pacientes son incorporados al programa. No registramos óbitos en el segundo año de experiencia.

Los pilares de los protocolos de recuperación acelerada se basan en la educación constante tanto del paciente incluido como de los especialistas intervenientes, convirtiendo a estos proyectos en un trabajo multidisciplinario con necesidad de auditorías y revisiones constantes y con dificultades en su aplicación. Es útil rescatar que cada institución, y sobre todos a aquellas pertenecientes a países en desarrollo deben adecuar los postulados internacionales a la realidad institucional y social del ámbito al que pertenecen. El factor organizacional es fundamental, como lo demuestra el trabajo publicado por Uriburu et al. ${ }^{6}$ en nuestro medio, con tasas de adherencias muy dispares según la intervención de cuidados perioperatorios que se analice.

A su vez, la aplicación de medidas que rompen los paradigmas tradicionales conservadores puede generar falta de adherencia por parte del equipo quirúrgico y anestesiólogos. Sin embargo hoy en día la literatura internacional cuenta con innumerables trabajos que abalan su implementación $2,11,16,17$. 
La preparación rica en carbohidratos recomendada por los consensos internaciones fue formulada por el equipo de nutrición de la institución ante la falta de ingreso de los mismos al mercado nacional, respetando los componentes esenciales de las mismas. Para tal fin se creó una bebida a base de bebida de rehidratación oral (Gaterode $\AA$ ), polimerosa y potasio vía oral como se especifica en la tabla 1. El paciente fue educado para cumplir con la ingesta de los mismos hasta 2 horas previas a la intervención quirúrgica.

Respecto a la preparación mecánica, tema de gran debate internacional, hoy en día la asociación americana de cirugía ${ }^{18}$ plantea que la preparación mecánica en conjunto con antibioticoterapia via oral es la mejor alternativa para prevenir fistulas anastomoticas e infección de herida quirúrgica. En nuestro caso hemos decidido la preparación solo en pacientes destinados a cirugía colonica izquierda y rectal, con una preparación reducida y domiciliaria que permite mantener las ventajas mencionadas de la misma pero con menor riesgo de deshidratación y desequilibrio hidroelectrolítico. En nuestra casuística no hemos detectado dicha complicación, por lo cual consideramos que podría conventirse en un futuro en una alternativa válida.

La edad avanzada, las comorbilidades relacionadas a patologías descompensadas, la malnutrición y los tumores malignos en estadios avanzados son factores de riesgo para una mayor morbilidad postoperatoria y para demoras en el alta, motivo por el cual fueron excluidos del presente trabajo. Sin embargo una vez lograda la correcta conjunción entre los distintos actores

intervenientes paradójicamente este grupo de pacientes son quienes más se benefician de este tipo de protocolos ${ }^{15,19,20}$.

Nuestro trabajo tiene limitaciones. Si bien es prospectivo, la selección de pacientes de manera consecutiva y no randomizada, menores de 80 años y con bajo riesgo según el ASA pueden haber influido en los resultados. Sin embargo la mejora lograda en los cuidados perioperatorios por todo el equipo interviniente nos permite inferir las conclusiones.

Nuestra serie demuestra que los protocolos de recuperación acelerada son un abordaje seguro y eficaz, con alta precoz y baja tasas de complicaciones, siempre y cuando se adecuen los postulados internacionales a la realidad de cada institución y región.

\section{Limitaciones de responsabilidad}

Los autores aceptan las limitaciones de responsabilidad de la revista, asumiendo cualquier requerimiento asociado a la publicación del presente trabajo.

\section{Originalidad del trabajo}

El presente trabajo es original y no forma parte de otra publicación o trabajo.

\section{Fuentes de apoyo}

No se contó con apoyo financiero externo.

\section{Derecho publicación y traducción}

Los autores cedemos el derecho de autor a la Universidad Nacional de Córdoba para publicar en la RFCMC y realizar la traducción en inglés.

\section{Bibliografía}

1. Kehlet $H$, Wilmore DW. Multimodal strategies to improve surgical outcome. Am J Surg. 2002;183(6):630-641.

2. Kehlet $H$, Wilmore DW. Evidence-based surgical care and the
evolution of fast-track surgery. Ann Surg. 2008;248(2):189-198.

3. Zargar-Shoshtari K, Hill AG. Optimization of perioperative care for colonic surgery: a review of the evidence. ANZ J Surg. 2008;78(1-2):13-23.

4. Lassen K, Soop M, Nygren J, et al. Consensus review of optimal perioperative care in colorectal surgery: Enhanced Recovery After Surgery (ERAS) Group recommendations. Arch Surg. 2009;144(10):961-969. doi:10.1001/archsurg.2009.170.
5. Zargar-Shoshtari K, Connolly AB, Israel LH, Hill AG. Fast-track surgery may reduce complications following major colonic surgery. Dis Colon Rectum. 2008;51(11):1633-1640.

6. Patrón Uriburu, Cillo M, Estefanía D, Ruiz H, Fernandez $L$ et al. Enhanced Recovery After Surgery ( ERAS ) Implementation of a Protocol in Laparoscopic Colectomy, Initial Experience in a Single Institution in Argentina. Gastroenterol Hepatol Open Access. 2016;4(3).

7. Dindo D, Demartines N, Clavien P-A. Classification of Surgical Complications: A New Proposal With Evaluation in a Cohort of 6336 Patients and Results of a Survey. Ann Surg. 2004;240(2):205-213.

8. Clavien PA, Barkun J, de Oliveira ML, et al. The Clavien-Dindo classification of surgical complications: five-year experience. Ann Surg. 2009;250(2):187-196.

9. Bruce J, Krukowski ZH, Al-Khairy G, Russell EM, Park KG. Systematic review of the definition and measurement of anastomotic leak after gastrointestinal surgery. $\mathrm{Br} J$ Surg. 2001;88(9):1157-1168.

10. Rahbari NN, Weitz J, Hohenberger W, et al. Definition and grading of anastomotic leakage following anterior resection of the rectum: a proposal by the International Study Group of Rectal Cancer. Surgery. 2010;147(3):339-351.

11. Singh PP, Hill AG. Fast-track elective colectomy: single-surgeon experience of 100 consecutive cases. N Z Med J. 2013;126(1369):8-15.

12. Aarts M-A, Okrainec A, Glicksman A, Pearsall E, Victor JC, McLeod RS. Adoption of enhanced recovery after surgery (ERAS) strategies for colorectal surgery at academic teaching hospitals and impact on total length of hospital stay. Surg Endosc. 2012;26(2):442-450.

13. Andersen J, Hjort-Jakobsen D, Christiansen PS, Kehlet $H$. Readmission rates after a planned hospital stay of 2 versus 3 days in fast-track colonic surgery. Br J Surg. 2007;94(7):890893.

14. Luglio G, De Palma GD, Tarquini $R$, et al. Laparoscopic colorectal surgery in learning curve: Role of implementation of a standardized technique and recovery protocol. A\&\#xa0;cohort study. Ann Med Surg. 2017;4(2):89-94.

15. Delaney CP, Fazio VW, Senagore AJ, Robinson $B$, Halverson $A L$, Remzi FH. "Fast track" postoperative management protocol for patients with high co-morbidity undergoing complex abdominal and pelvic colorectal surgery. $\mathrm{Br} J$ Surg. 2001;88(11):1533-1538.

16. Alvarez MP, Foley KE, Zebley DM, Fassler SA. Comprehensive enhanced recovery pathway significantly reduces postoperative length of stay and opioid usage in elective laparoscopic colectomy. Surg Endosc. 2015;29(9):2506-2511.

17. Bakker N, Cakir H, Doodeman HJ, Houdijk APJ. Eight years of experience with Enhanced Recovery After Surgery in patients with colon cancer: Impact of measures to improve adherence. Surgery. 2015;157(6):1130-1136.

18. Kiran RP, Murray ACA, Chiuzan C, Estrada D, Forde $K$. Combined preoperative mechanical bowel preparation with oral antibiotics significantly reduces surgical site infection, anastomotic leak, and ileus after colorectal surgery. Ann Surg. 2015;262(3):415-416.

19. Bardram L, Funch-Jensen $P$, Kehlet $H$. Rapid rehabilitation in elderly patients after laparoscopic colonic resection. $\mathrm{Br} J$ Surg. 2000;87(11):1540-1545.

20. Forsmo HM, Erichsen $C$, Rasdal $A$, Körner $H$, Pfeffer $F$. Enhanced Recovery After Colorectal Surgery (ERAS) in Elderly Patients Is Feasible and Achieves Similar Results as in Younger Patients. Gerontol Geriatr Med. 2017 JanDec;3:2333721417706299. doi: 10.1177/2333721417706299. 